\title{
Ultrafast insulator-to-metal phase transition as a switch to measure the spectrogram of a supercontinuum light pulse
}

\author{
Federico Cilento ${ }_{3}^{1,2}$ Claudio Giannetti, ${ }^{3, a)}$ Gabriele Ferrini, ${ }^{3}$ Stefano Dal Conte ${ }^{4}$ \\ Tommaso Sala, ${ }^{3}$ Giacomo Coslovich, ${ }^{1,2}$ Matteo Rini, ${ }^{5}$ Andrea Cavalleri, ${ }^{6,3}$ and \\ Fulvio Parmigiani ${ }^{1,8}$ \\ ${ }^{1}$ Department of Physics, Università degli Studi di Trieste, Trieste I-34127, Italy \\ ${ }^{2}$ Laboratorio Nazionale TASC, AREA Science Park, Basovizza Trieste I-34012, Italy \\ ${ }^{3}$ Department of Physics, Università Cattolica del Sacro Cuore, Brescia I-25121, Italy \\ ${ }_{5}^{4}$ Department of Physics A. Volta, Università degli Studi di Pavia, Pavia I-27100, , Italy \\ ${ }^{5}$ Materials Sciences Division, Lawrence Berkeley National Laboratory, Berkeley, California 94720, USA \\ ${ }^{6}$ Condensed Matter Division, Max Planck Research Department for Structural Dynamics, University \\ of Hamburg, 22607 Hamburg, Germany \\ ${ }^{7}$ Department of Physics, Clarendon Laboratory, University of Oxford, OX1 3PU Oxford, United Kingdom \\ ${ }^{8}$ Sincrotrone Trieste S.C.p.A., Basovizza, Trieste I-34012, Italy
}

(Received 20 October 2009; accepted 10 December 2009; published online 11 January 2010)

\begin{abstract}
In this letter we demonstrate the possibility to determine the temporal and spectral structure (spectrogram) of a complex light pulse exploiting the ultrafast switching character of a nonthermal photoinduced phase transition. As a proof, we use a $\mathrm{VO}_{2}$ multifilm, undergoing an ultrafast insulator-to-metal phase transition when excited by femtosecond near-infrared laser pulses. The abrupt variation in the multifilm optical properties, over a broad infrared/visible frequency range, is exploited to determine, in situ and in a simple way, the spectrogram of a supercontinuum pulse produced by a photonic crystal fiber. The determination of the structure of the pulse is mandatory to develop pump-probe experiments with frequency resolution over a broad spectral range (700-1100 nm). (C) 2010 American Institute of Physics. [doi:10.1063/1.3291105]
\end{abstract}

Recently, it has become possible to generate broadband continuum, extending from 450 to $1600 \mathrm{~nm}$ with a nearly flat spectral intensity, from few nanojoule pulses produced by a standard $120 \mathrm{fs} 800 \mathrm{~nm}$ Ti:sapphire oscillator. The key elements in continuum generation are the newly developed microstructured photonic-crystal fibers (PCF), engineered to be nearly dispersion-free at particular frequencies in the nearinfrared/visible range. Nonlinear interactions between an infrared laser pulse propagating into the fiber and the silica core generate a broadband nontransform-limited pulse output with unknown residual spectral chirp. ${ }^{1}$ Since supercontinuum (SC) is emerging as a new tool for time-resolved spectroscopic techniques with broadband frequency resolution, ${ }^{2}$ it is mandatory to find simple methods to fully characterize the temporal and spectral structure (spectrogram) of complex light pulses.

Usually, nonlinear gating techniques, such as frequencyresolved optical gating (FROG), ${ }^{3}$ are used to characterize continuum optical pulses. ${ }^{4}$ These techniques require a phase matchable and transparent nonlinear optical medium across the entire bandwidth of the continuum pulse. The problems in obtaining phase-matching conditions over a broad spectral range, with a single crystal, are generally solved by angledithering techniques. ${ }^{3}$

Although it is possible to use these nonlinear techniques to fully characterize the continuum, practical problems in a typical pump and probe set-up (see Fig. 1) demand for an ad hoc solution. ${ }^{5}$ The need to characterize the continuum pulses in situ, having access only to their reflection from a sample held inside a cryostat, possibly without the need of phasematching conditions, prompted us to consider the possibility

${ }^{a)}$ Electronic mail: c.giannetti@dmf.unicatt.it. of a solid-state ultrafast optical switch as a gating element. A thin $\mathrm{VO}_{2}$ film, when excited with a near infrared ultrashort laser pulse, has the remarkable property of undergoing a photoinduced insulator-to-metal phase transition, ${ }^{6,7}$ resulting in an ultrafast change of the reflectivity over a broad frequency range. In addition, the switching character of the system, due to the fact that the recovery time (10-100 ns) of the excited film is longer than the pulse duration, is also useful to check for multiple-pulses in the laser output.

In this letter, we demonstrate the application of a $\mathrm{VO}_{2}$ thin-film multilayer sample as an ultrafast optical switch to perform a frequency and time characterization of a PCFgenerated ultrabroad and ultrashort laser pulse, through a time resolved transient optical spectroscopy in a pump/probe configuration. The major advantages of this technique are that no phase matching is needed, the fluence threshold for the process is reasonably low $\left[\sim 250 \mu \mathrm{J} / \mathrm{cm}^{2}\right.$ (Ref. 8) $]$ to achieve, and the multilayer can be easily placed at the

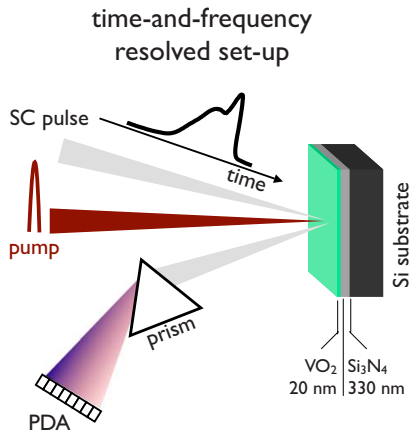

$\mathrm{VO}_{2}$-based gating technique

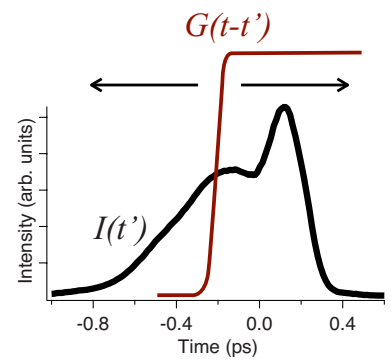

FIG. 1. (Color online) A sketch of the experimental set-up and of the $\mathrm{VO}_{2}$-based gating technique is reported. 
sample position without altering the optical design of the system, arranged to work in a reflectivity configuration.

A broadband electromagnetic field pulse with a complex temporal structure, i.e., $E(t)=[I(t)]^{1 / 2} e^{i \phi(t)}$ $=\int[\widetilde{I}(\omega)]^{1 / 2} \mathrm{e}^{i \Phi(\omega)} \mathrm{e}^{i \omega t} d \omega / 2 \pi[I(t)$ being the slowly-varying intensity envelope], is characterized by the variation in the frequency with time (chirp) $\Omega(t)=-d \phi(t) / d t$ or, in the frequency domain, by $T(\omega)=-d \Phi(\omega) / d \omega$, i.e., the group delay of a particular slice of the spectrum at frequency $\omega$. The complete reconstruction of the time-frequency characteristics of the light pulse is called spectrogram and it is mathematically given by

$$
S(\omega, t)=\left|\int_{-\infty}^{\infty} E\left(t^{\prime}\right) G\left(t-t^{\prime}\right) \mathrm{e}^{-i \omega t^{\prime}} d t^{\prime}\right|^{2},
$$

where $G\left(t-t^{\prime}\right)$ is a gate function, whose temporal delay $t$ $-t^{\prime}$ can be varied. In standard nonlinear FROG techniques ${ }^{3}$ an ultrashort laser pulse is used as the temporal gate, while a thin slice of the spectrum is filtered by the sum-frequency process on the nonlinear crystal. In our approach, the gate function is replaced by a steplike function represented by the $\mathrm{VO}_{2}$ film reflectivity variation, triggered by a $\sim 120$ fs $-800 \mathrm{~mm}$ pump pulse (see Fig. 1). The activation time $t-t^{\prime}$ is delayed through the duration of the SC pulse simply varying the relative optical path between the pump and probe pulses. In this configuration, the time resolution of the measured spectrogram is given by the time needed to switch the $\mathrm{VO}_{2}$ optical properties, which is shorter than the 120 fs pulse duration. ${ }^{9}$ Since the $\mathrm{VO}_{2}$ reflectivity variation extends over a broad frequency range, the spectral resolution is determined by the width of each slice of the spectrum acquired by the detection system.

The optical pump-probe setup is based on a cavitydumped Ti:sapphire oscillator. The output is a train of 800 $\mathrm{nm}-120 \mathrm{fs}$ pulses with an energy of $40 \mathrm{~nJ} /$ pulse. The $12 \mathrm{~cm}$ long PCF with a $1.6 \mu \mathrm{m}$ core is pumped with $5 \mathrm{~nJ} /$ pulse focused into the core by an aspherical lens. The SC-probe output is collimated and refocused on the sample through achromatic doublets in the near-IR/visible range. The $800 \mathrm{~nm}$ oscillator output (pump) and SC beam (probe), orthogonally polarized, are noncollinearly focused onto the sample, checking their superposition and dimensions $(40 \mu \mathrm{m}$ for the IRpump and $20 \mu \mathrm{m}$ for the SC probe) with a charge-coupled device camera. The pump fluence is about $3 \mathrm{~mJ} / \mathrm{cm}^{2}$, well above the $\mathrm{VO}_{2}$ phase transition threshold. ${ }^{8}$ The reflected probe beam is spectrally dispersed through a SF11 equilateral prism and imaged on a 128 pixel linear photodiode array (PDA), capturing the 700-1100 $\mathrm{nm}$ spectral region. A spectral slice, whose width ranges from $2 \mathrm{~nm}$ at $700 \mathrm{~nm}$ to $6 \mathrm{~nm}$ at $1100 \mathrm{~nm}$, is acquired by each pixel of the array, corresponding to a constant temporal resolution of $\sim 120$ fs. The probe beam is sampled before the interaction with the pump and used as a reference for the SC intensity. The outputs of the two PDAs are acquired through a $22 \mathrm{bit} / 2 \mathrm{MHz}$ fast digitizer, and are divided pixel by pixel to compensate the SC intensity fluctuations, obtaining a signal to noise ratio of the order of $10^{-4}$ acquiring 2000 spectra $(\sim 1 \mathrm{~s}$ integration time, cf. Ref. 10). The differential reflectivity signal is obtained modulating the pump beam with a mechanical chopper and performing the difference between unpumped and pumped spectra. The multilayer sample is a $20 \mathrm{~nm} \mathrm{VO}_{2}$ thin

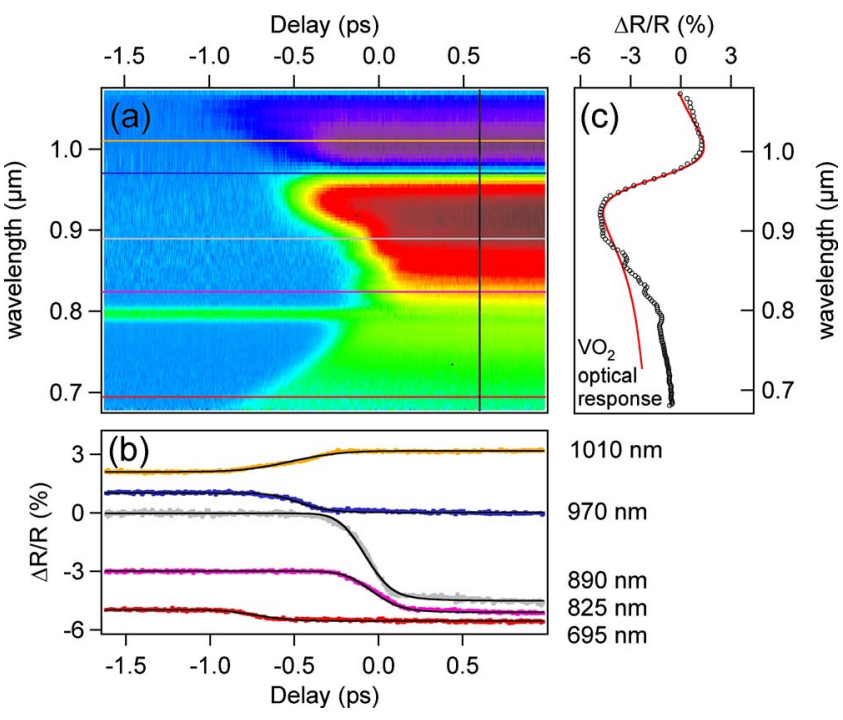

FIG. 2. (Color online) (a) The time-and-frequency resolved reflectivity variation, measured on the $\mathrm{VO}_{2}$ multi-film sample, is shown. (b) Time traces at different wavelengths are reported. The solid lines are the fit to the data, as described in the text. (c) The frequency-resolved reflectivity variation at $t-t^{\prime}=0.6 \mathrm{ps}$ is reported. The solid line is the reflectivity variation mimicked replacing the $\mathrm{VO}_{2}$ insulating dielectric function with the metallic one.

film on a $330 \mathrm{~nm} \mathrm{Si}_{3} \mathrm{~N}_{4}$ buffer layer, deposited on a silicon substrate.

In Fig. 2(a) we report the frequency resolved $\mathrm{VO}_{2}$ reflectivity variation, measured as a function of the delay between the supercontinuum pulse and the $\mathrm{VO}_{2}$ switching, triggered by the pump pulse. A decrease in the reflectivity of a few percent is measured below $970 \mathrm{~nm}$, whereas, above this value, the reflectivity variation becomes positive. The onset of the reflectivity variation exhibits a parabolic shape with wavelength, related to the parabolic group delay variation with wavelength typical of PCF-generated supercontinuum pulses. ${ }^{4}$ The flat signal measured at $800 \mathrm{~nm}$ is due to a low intensity postpulse produced by the cavity and entering in the PCF about 18 ps after the main pulse. The small intensity $(<1 \%$ of the main pulse) of the postpulse prevents it from nonlinearly interacting with the fiber and broadening its spectrum. We underline that the switching character of $\mathrm{VO}_{2}$ reveals the presence of postpulses even if the investigated temporal window is small as compared to the delay between the main and the secondary pulses. In Fig. 2(b) different time-traces at specific wavelengths are shown. A convolution between a step function centered at $t-t^{\prime}$, representing the $\mathrm{VO}_{2}$ reflectivity variation, and a Gaussian is fitted to the time-traces [solid lines in Fig. 2(b)]. The Gaussian takes into account both the temporal resolution (pulse width and $\mathrm{VO}_{2}$ response) and the time width of the wavepacket centered at the frequency $\omega$ detected by the pixel. The extracted $t-t^{\prime}$ values and Gaussian widths represent the delays and the temporal widths, respectively, of the particular slices of the spectrum corresponding to different pixels of the detector.

To give more insights into the origin of the ultrafast variation of the $\mathrm{VO}_{2}$ optical properties, in Fig. 2(c) we report the frequency-resolved reflectivity variation, measured at a delay of $+0.6 \mathrm{ps}$ from the pump pulse and representing the optical response of the device. The complex reflectivity variation exhibited by the $\mathrm{VO}_{2}$ sample is the result of both the occurrence of the insulator-to-metal phase transition and the multiple internal reflections at the multifilm interfaces. 

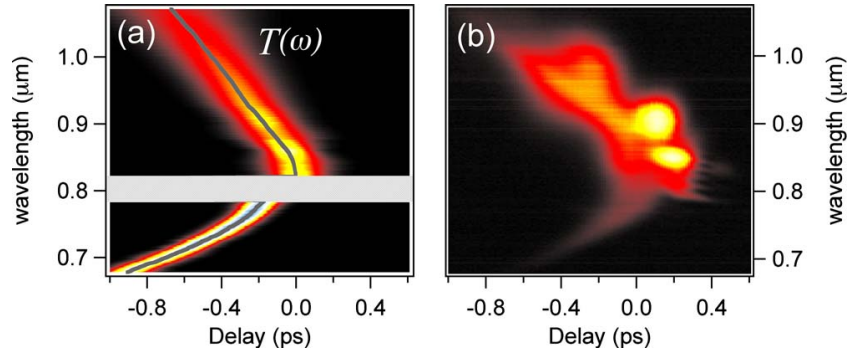

FIG. 3. (Color online) (a) The $T(\omega)$ of the SC pulse, reconstructed through the $\mathrm{VO}_{2}$-based technique described in the text, is reported. The solid line is the $t-t^{\prime}$ delay between the step function, mimicking the $\mathrm{VO}_{2}$ switch opening, and the probe pulse. (b) The SC spectrogram, measured through a standard XFROG technique, is shown.

The measured reflectivity variation has been qualitatively reproduced [solid line in Fig. 2(c)], taking into account the sample geometry and replacing the dielectric constant of the $\mathrm{VO}_{2}$ insulating phase with that of the metallic phase. In particular, the insulating dielectric function is described by four Lorentz oscillators with frequencies at 1.25, 2.8, 3.6, and $4.9 \mathrm{eV}^{11}$ whereas the optical properties of the conducting phase are mimicked substituting the $1.25 \mathrm{eV}$ band-gap related oscillator with a Drude term, representing the metallic free electrons, and slightly varying the energies, plasma frequencies and damping of the other oscillators, to take into account the shift of the $\mathrm{O} 2 p$ and $\mathrm{V} 3 d$ bands, related to the change in the crystal structure. ${ }^{12}$ The refraction indexes of the other materials constituting the multifilm sample have been taken from Ref. 13. The fact that the photoinduced variation in the sample optical response extends throughout a broad spectral range, confirms the choice of this system as an ultrafast optical switch.

In Fig. 3 we compare the $T(\omega)$ of the SC pulse [Fig. 3(a)], reconstructed through the procedure described above, to the trace [Fig. 3(b)] obtained through a standard crosscorrelation FROG technique (XFROG). ${ }^{4}$ The XFROG trace has been obtained performing a cross-correlation between the $800 \mathrm{~nm}$ pump pulse and the SC probe pulse and recording the sum frequency intensity, generated in the angledithered BBO crystal, through a UV spectrometer. While the $\mathrm{VO}_{2}$-based method exhibits a poorer time-resolution, as compared to the XFROG technique, all the main temporal features of the SC pulse can be reconstructed. In particular, the parabolic group delay variation with wavelength of the PCF-generated pulse can be satisfactorily measured. The limitations in the time resolution are mainly related to the pump pulse time-width, to the fitting procedures and to the velocity of the impulsive variation in the $\mathrm{VO}_{2}$ optical properties, which is limited, in the present case, by the sample quality and by the intrinsic finite time for the $\mathrm{VO}_{2}$ switching. Improving the quality of the device and optimizing the fitting procedures will appreciably increase the resolution of this solid-state based technique. Alternatively, different materials with a more rapid switching time should be considered. ${ }^{14}$

Finally, the $\mathrm{VO}_{2}$-based technique exhibits some fundamental advantages as compared to the XFROG which are as follows: (i) the SC pulse characterization can be performed in the reflectivity configuration, without the need of phasematching conditions. This permits to characterize SC pulses in situ, close to samples where time-and-frequency reflectivity experiments are performed, without the need to replace the sample holder with an angle-dithered BBO crystal. (ii) Multiple-pulses, severely affecting ultrafast laser applications, can be easily detected without the need of timeconsuming scanning of the entire temporal window between two subsequent pulses.

We acknowledge the comments from C. Manzoni. F.C., G.C., and F.P. acknowledge the support of the Italian Ministry of University and Research under Grant Nos. FIRBRBAP045JF2 and FIRB-RBAP06AWK3.

${ }^{1}$ J. M. Dudley, G. Genty, and S. Coen, Rev. Mod. Phys. 78, 1135 (2006).

${ }^{2}$ J. Léonard, N. Lecong, J.-P. Likforman, O. Crégut, S. Haacke, P. Viale, P. Leproux, and V. Couderc, Opt. Express 15, 16124 (2007).

${ }^{3} \mathrm{R}$. Trebino, Frequency-Resolved Optical Gating: The Measurement of Ultrashort Laser Pulses (Springer, New York, 2002).

${ }^{4}$ J. M. Dudley, X. Gu, L. Xu, M. Kimmel, E. Zeek, P. O'Shea, R. Trebino, S. Coen, and R. S. Windeler, Opt. Express 10, 1215 (2002).

${ }^{5}$ E. K. Tien, X. Z. Sang, F. Qingí, Q. Song, and O. Boyraz, Appl. Phys. Lett. 95, 051101 (2009).

${ }^{6}$ A. Cavalleri, C. Toth, C. W. Siders, J. A. Squier, F. Raksi, P. Forget, and J. C. Kieffer, Phys. Rev. Lett. 87, 237401 (2001).

${ }^{7}$ A. Cavalleri, H. H. W. Chong, S. Fourmaux, T. E. Glover, P. A. Heimann, J. C. Kieffer, B. S. Mun, H. A. Padmore, and R. W. Schoenlein, Phys. Rev. B 69, 153106 (2004).

${ }^{8}$ M. Rini, Z. Hao, R. W. Schoenlein, C. Giannetti, F. Parmigiani, S. Fourmaux, J. C. Kieffer, A. Fujimori, M. Onoda, S. Wall, and A. Cavalleri, Appl. Phys. Lett. 92, 181904 (2008).

${ }^{9}$ A. Cavalleri, T. Dekorsy, H. H. W. Chong, J. C. Kieffer, and R. W. Schoenlein, Phys. Rev. B 70, 161102 (2004).

${ }^{10}$ D. Polli, L. Luer, and G. Cerullo, Rev. Sci. Instrum. 78, 103108 (2007).

${ }^{11}$ H. W. Verleur, A. S. Barker, and C. N. Berglund, Phys. Rev. 172, 788 (1968)

${ }^{12}$ A. Cavalleri, M. Rini, H. H. W. Chong, S. Fourmaux, T. E. Glover, P. A. Heimann, J. C. Kieffer, and R. W. Schoenlein, Phys. Rev. Lett. 95, 067405 (2005).

${ }^{13}$ The optical constants of $\mathrm{Si}$ and $\mathrm{Si}_{3} \mathrm{~N}_{4}$ are taken from www.luxpop.com.

${ }^{14}$ D. Polli, M. Rini, S. Wall, R. W. Schoenlein, Y. Tomioka, Y. Tokura, G. Cerullo, and A. Cavalleri, Nature Mater. 6, 643 (2007). 